\title{
The pathophysiology of diastolic heart failure
}

\section{Thanh Trung Phan and Michael Frenneaux*}

\author{
Address: School of Medicine and Dentistry, University of Aberdeen, Polwarth Building, Foresterhill, Aberdeen, AB25 2ZD, Scotland \\ *Corresponding author: Michael Frenneaux (m.p.frenneaux@abdn.ac.uk) \\ FI000 Biology Reports 2010, 2:16 (doi:10.34I0/B2-16)
}

The electronic version of this article is the complete one and can be found at: http://f1000.com/reports/biology/content/2/I6

\begin{abstract}
Whilst resting disturbances of both diastolic and long-axis systolic function are observed in patients with heart failure who have normal left ventricular ejection fraction, recent evidence suggests that dynamic disturbances in cardiac function occur during exercise. A paradoxical slowing of left ventricular active relaxation during exercise limits cardiac filling and therefore stroke volume and appears to be due to the combination of cardiac energetic impairment and disturbed ventricularvascular coupling.
\end{abstract}

\section{Introduction and context}

Many consider heart failure with preserved ejection fraction (HFpEF) to be a disorder of diastolic function (hence the term 'diastolic heart failure') [1], whilst others believe that it may be due to a combination of diastolic abnormalities with subtle disturbances of systolic function that are insufficient to reduce left ventricular ejection fraction (LVEF) [2]. In this review, HFpEF refers to heart failure patients with an LVEF of greater than $50 \%$ $[3,4]$.

\section{Resting left ventricular stiffness in HFpEF}

Diastolic function is influenced by passive elastic properties of the left ventricle, by the highly energy-dependent process of active relaxation, and by the atrial contribution to filling. Zile et al. [5] demonstrated that patients with HFpEF had abnormal left ventricular (LV) relaxation and increased resting LV stiffness with a shift in the diastolic pressure-volume relationship upwards and to the left. However, other studies have shown that resting ventricular stiffness is not invariably increased in patients with HFpEF [6]. Increased myocardial mass or changes in the extra-myocardial collagen network [1] (increased collagen content and increased collagen cross-linking) may contribute to increased LV passive diastolic stiffness at rest [5]. A study by van Heerebeek et al. [7] examined differences in titin isoform expression between patients with systolic and 'diastolic heart failure'. Titin is a large cytoskeletal protein that contributes to resting stiffness of the myocardium [8]. A shift toward the stiffer N2B isoform was observed in the diastolic heart failure group and toward the longer N2BA isoform in the systolic heart failure group when compared with previously published results from healthy controls [7]. A shift to expression of the shorter N2B isoform in response to increased arterial stiffness would increase 'contractility' (to compensate for increased aortic impedance) at the price of increased LV systolic and diastolic stiffness. More recently, myocardial biopsies from patients with aortic stenosis and patients with heart failure suggest that, rather than just a shift in titin isoforms, other mechanisms such as relative hypophosphorylation of the stiffer N2B titin isoform were a possible cause of increased LV stiffness in failing myocardium [9].

\section{Resting large-artery stiffness and ventricular-vascular coupling}

The interaction between the heart and the systemic vasculature, termed ventricular-vascular coupling (VVC), is essential for the heart to achieve maximal cardiac work, power and chamber efficiency while maintaining physiological blood pressures and cardiac outputs [10]. VVC is the ratio of arterial elastance $\left(\mathrm{E}_{\mathrm{a}}\right)$ to end-systolic elastance $\left(\mathrm{E}_{\mathrm{es}}\right)$. LV end-systolic elastance (stiffness) $\left(\mathrm{E}_{\mathrm{es}}\right)$ is calculated from the slope of the end-systolic pressurevolume relationship. Arterial elastance (stiffness) $\left(E_{a}\right)$ is 
a measure of impedance and is determined by the ratio of systolic pressure to stroke volume.

Ventricular-vascular interaction is important in the context of HFpEF because of its important effects on diastolic filling [11]. In patients with HFpEF, the resting VVC is lower than in younger individuals [12] but similar to asymptomatic hypertensive elderly patients [13] and falls within a range in which cardiac work and efficiency are not compromised [14]. However, although the resting VVC ratio is within the physiological range, the absolute values of $E_{e s}$ and $E_{a}$ are considerably elevated, indicating increased arterial and ventricular systolic stiffness and this becomes important during exercise (see below).

\section{Systolic, diastolic and vascular function during exercise}

In young healthy subjects, exercise is associated with an increase in contractility and in the rate of $\mathrm{LV}$ active relaxation, although the latter is attenuated with increasing age [15]. In HFpEF, these physiological changes during exercise are profoundly deranged and this appears to be central to the pathophysiology of the disorder. In a small study by Kitzman et al. [16], HFpEF patients underwent invasive cardiopulmonary exercise testing. HFpEF patients exhibited a shift of the LV enddiastolic pressure-volume relationship upward and to the left at rest, and during exercise increases in LV filling pressure were not accompanied by increases in enddiastolic volume index, indicating a limitation to $\mathrm{LV}$ filling during exercise and a failure of the Frank-Starling mechanism [16]. More recently, a study conducted by Kawaguchi et al. [6] reported a dynamic impairment of LV active relaxation during isometric (handgrip) exercise in a group of HFpEF patients.

These findings suggest a potentially attractive link between increased large-artery stiffness and exerciseinduced diastolic dysfunction. Animal studies have demonstrated that a large acute increase in afterload in healthy hearts of rabbits resulted in a marked slowing of active relaxation and impaired LV diastolic filling [17]. However, acute increase in afterload required to cause a slowing of active relaxation may be much less in a diseased heart compared with a healthy heart. The concept of 'relative load', which represents the ratio of systolic LV pressure to isovolumetric LV pressure [18], allows for the possibility that an increase in afterload required to cause a slowing of active relaxation may be much less in a diseased heart compared with a healthy heart. A similar systolic LV pressure represents a higher relative load in the failing heart than in the normal heart. When relative load is low, afterload reserve is still available, allowing the heart to face increased afterload without slowing of LV active relaxation. When relative load is high, afterload mismatch [19] occurs and a pronounced slowing of LV active relaxation is observed [18]. Other extra-cardiac factors that may contribute to the pathophysiology of HFpEF include volume overload in conditions such as anaemia, renal dysfunction and obesity [20]. In addition, previous data in an animal model have indicated that neurohormonal activation with intravascular volume expansion following relatively small degrees of ischaemic myocardial injury can lead to elevated LV end-diastolic pressure in the absence of diastolic dysfunction or reductions in maximal $\mathrm{dP} / \mathrm{dt}$ (rate of rise of LV pressure) or EF [21].

\section{Major recent advances}

Borlaug and colleagues [22] recently reported that hypertensive patients with or without HFpEF had increased arterial and LV stiffness but similar VVC at rest. However, despite the apparently 'preserved' EF, patients with HFpEF had depressed cardiac contractility compared with asymptomatic hypertensive subjects [22]. This was consistent with other studies that had used tissue Doppler imaging and had demonstrated the presence of diastolic or systolic dyssynchrony or both in patients with HFpEF $[23,24]$.

A study by Westermann et al. [25], involving pressurevolume loop analysis with and without atrial pacing in 70 HFpEF patients and 20 matched controls, found that enhanced LV stiffness can result in increased enddiastolic pressure during handgrip exercise. In addition, during atrial pacing, pacing with HFpEF displayed decreased stroke volume, but it should be noted that these patients were resting in a supine position and thus to artificially increase heart rate by atrial pacing might not reflect true physiological exercise conditions. At the very least, these data suggest that LV stiffness can modulate cardiac function in HFpEF patients. Another group, also using pressure-volume loop analysis, demonstrated that in HFpEF patients there was indeed increased LV passive stiffness and prolonged LV relaxation at rest and, interestingly, that during atrial pacing, $\mathrm{LV}$ relaxation reserve was limited but the cardiac contractility was normal. The net result was a blunted cardiac output during atrial pacing [26]. Increased LV stiffness in HFpEF patients may reflect not only increased myocardial mass and changes in extra-myocardial collagen (e.g., titins) but also LV fibrosis as demonstrated by Martos and colleagues [27], who found marked serological evidence of active fibrotic processes in patients with HFpEF.

Using echocardiographic techniques, our group recently demonstrated that, at rest, LV torsion and strain patterns 
in HFpEF patients were similar to those in age-related controls [28] but that during exercise HFpEF patients had reduced systolic and diastolic function as well as evidence of delayed LV untwisting and LV suction [29]. In a separate study, using radionuclide ventriculography, we studied $37 \mathrm{HFpEF}$ patients and 20 control subjects during cycle exercise and demonstrated that HFpEF patients had marked disturbances of VVC and of both systolic and diastolic function which appeared to be responsible for exercise limitation. LV active relaxation was paradoxically slowed. $E_{a}$ fell less and $E_{e s}$ increased much less during exercise than in age-matched controls, the latter indicating a failure of contractile reserve [30]. The impaired LV diastolic filling during exercise may be partly compensated by increased left atrial contribution during the final stages of diastolic filling [31] until atrial failure and eventually atrial fibrillation supervene later in the natural history of the disease.

A key coupler of load-dependent $\mathrm{LV}$ relaxation is troponin I-protein kinase A (TnI-PKA) phosphorylation. This energy-dependent process of phosphorylation of troponin I by PKA decreases myofibrillar calcium sensitivity and increases the rate at which calcium dissociates from troponin $\mathrm{C}$ and this can lead to an increased rate of $\mathrm{LV}$ relaxation by increasing the rate of thin filament deactivation. Using magnetic resonance spectroscopy, we recently demonstrated that HFpEF patients had reduced myocardial energetic reserve at rest (decreased phosphocreatine/adenosine triphosphate [PCr/ATP] ratio), and this might explain why these HFpEF patients are particularly prone to impaired LV active relaxation during exercise and impaired contractile reserve [30].

The cause for this resting energy deficit may relate to insulin resistance, to impaired mitochondrial function as a result of ageing, and to neuro-endocrine activation and aberrant substrate metabolism. In addition, a recent study in patients with hypertrophic cardiomyopathy found that reduced myocardial PCr/ATP correlated with the presence of fibrotic area in the myocardium of the left ventricle [32].

\section{Future directions}

There are consistent data demonstrating markedly abnormal diastolic responses to exercise in HFpEF. In our view, the detection of HFpEF patients cannot rely solely on resting parameters and we need to consider some form of exercise testing such as metabolic exercise testing or exercise radionuclide ventriculography or echocardiography scans in the future.

Future studies should begin to translate key pathophysiological findings in HFpEF to an effective clinical therapy. An effective therapy for HFpEF patients has yet to be developed. However, the finding that HFpEF patients have reduced myocardial energetic status may provide the rationale to assess the therapeutic value of 'metabolic agents' (e.g., perhexiline and trimatazidine) that increase cardiac energetic status by altering cardiac substrate use [33]. LV stiffness and fibrosis could be addressed by drugs such as aldosterone antagonists (e.g., eplerenone), which may help to alleviate aldosteroneinduced cardiac fibrosis, which causes increased stiffness, impaired LV relaxation and impaired LV filling.

\section{Abbreviations}

$\mathrm{E}_{\mathrm{a},}$ arterial elastance (stiffness); $\mathrm{E}_{\mathrm{es}}$ end-systolic elastance (stiffness); EF, ejection fraction; HFpEF, heart failure with preserved ejection fraction; LV, left ventricular; LVEF, left ventricular ejection fraction; $\mathrm{PCr} / \mathrm{ATP}$, phosphocreatine/ adenosine triphosphate; PKA, protein kinase A; VVC, ventricular-vascular coupling.

\section{Competing interests}

MF has received honoraria from Medronic (Minneapolis, MN, USA), St Jude Medical (St Paul, MN, USA), and Biotronik (Berlin, Germany) (amount of less than $\$ 10,000$ ) and has served as a consultant and advisory board member of Medronic, St Jude Medical, Menarini (Florence, Italy) and Biotronik (amount of less than $\$ 10,000)$ and on the speaker panel for Menarini (amount of less than $\$ 10,000$ ). TTP declares that he has no competing interests.

\section{References}

I. Zile MR, Brutsaert DL: New concepts in diastolic dysfunction and diastolic heart failure: Part I: diagnosis, prognosis, and measurements of diastolic function. Circulation 2002, I05: I387-93.

2. Yu CM, Lin H, Yang H, Kong SL, Zhang Q, Lee SW: Progression of systolic abnormalities in patients with 'isolated' diastolic heart failure and diastolic dysfunction. Circulation 2002, I05: I 195-201.

3. Vasan RS, Levy D: Defining diastolic heart failure: a call for standardized diagnostic criteria. Circulation 2000, I 0 I:2 I I8-2I.

4. Yturralde RF, Gaasch WH: Diagnostic criteria for diastolic heart failure. Prog Cardiovasc Dis 2005, 47:3।4-9.

5. Zile MR, Baicu CF, Gaasch WH: Diastolic heart failureabnormalities in active relaxation and passive stiffness of the left ventricle. N Engl J Med 2004, 350:1953-9.

6. Kawaguchi M, Hay I, Fetics B, Kass DA: Combined ventricular systolic and arterial stiffening in patients with heart failure and preserved ejection fraction: implications for systolic and diastolic reserve limitations. Circulation 2003, I07:7I4-20.

7. van Heerebeek L, Borbély A, Niessen HW, Bronzwaer JG, van der Velden J, Stienen GJ, Linke WA, Laarman GJ, Paulus WJ: Myocardial structure and function differ in systolic and diastolic heart failure. Circulation 2006, I I3:1966-73.

8. Lim CC, Sawyer DB: Modulation of cardiac function: titin springs into action. J Gen Physiol 2005, I 25:249-52.

9. Borbely A, Falcao-Pires I, van Heerebeek L, Hamdani N, Edes I, Gavina C, Leite-Moreira AF, Bronzwaer JGF, Papp Z, van der Velden J, Stienen GJM, Paulus WJ: Hypophosphorylation of the stiff N2B 
titin isoform raises cardiomyocyte resting tension in failing human myocardium. Circ Res 2009, 104:780-6.

10. Little WC, Cheng CP: Effect of exercise on left ventriculararterial coupling assessed in the pressure-volume plane. $\mathrm{Am} \mathrm{J}$ Physiol Heart Circ Physiol 1993, 264:1629-33.

II. Frenneaux M, Williams L: Ventricular-arterial and ventricularventricular interactions and their relevance to diastolic filling. Prog Cardiovasc Dis 2007, 49:252-62.

12. Chen CH, Nakayama M, Nevo E, Fetics BJ, Maughan WL, Kass DA: Coupled systolic-ventricular and vascular stiffening with age implications for pressure regulation and cardiac reserve in the elderly. J Am Coll Cardiol 1998, 32:1221-7.

13. Lam CSP, Roger VL, Rodeheffer RJ, Bursi F, Borlaug BA, Ommen SR, Kass DA, Redfield MM: Cardiac structure and ventricularvascular function in persons with heart failure and preserved ejection fraction from Olmsted County, Minnesota. Circulation 2007, I | 5: 1982.

14. De Tombe PP, Jones S, Burkhoff D, Hunter WC, Kass DA: Ventricular stroke work and efficiency both remain nearly optimal despite altered vascular loading. Am J Physiol Heart Circ Physiol 1993, 264:1817-24.

15. Nonogi H, Hess OM, Ritter M, Krayenbuehl HP: Diastolic properties of the normal left ventricle during supine exercise. $\mathrm{Br}$ Heart J 1988, 60:30-8.

16. Kitzman DW, Higginbotham MB, Cobb FR, Sheikh KH, Sullivan MJ: Exercise intolerance in patients with heart failure and preserved left ventricular systolic function: failure of the Frank-Starling mechanism. J Am Coll Cardiol 1991, I7:1065-72.

17. Leite-Moreira AF, Correia-Pinto J, Gillebert TC: Afterload induced changes in myocardial relaxation: a mechanism for diastolic dysfunction. Cardiovasc Res 1999, 43:344-53.

18. Gillebert TC, Leite-Moreira AF, De Hert SG: Relaxation-systolic pressure relation. A load-independent assessment of left ventricular contractility. Circulation 1997, 95:745-52.

19. Ross J Jr: Afterload mismatch and preload reserve: a conceptual framework for the analysis of ventricular function. Prog Cardiovasc Dis 1976, 18:255-64.

20. Maurer MS, Burkhoff D, Fried LP, Gottdiener J, King DL, Kitzman DW: Ventricular structure and function in hypertensive participants with heart failure and a normal ejection fraction: the Cardiovascular Health Study. J Am Coll Cardiol 2007, 49:972-8I.

21. He KL, Dickstein M, Sabbah HN, Yi GH, Gu A, Maurer M, Wei CM, Wang J, Burkhoff $D$ : Mechanisms of heart failure with well preserved ejection fraction in dogs following limited coronary microembolization. Cardiovasc Res 2004, 64:72-83.

22. Borlaug BA, Lam CSP, Roger VL, Rodeheffer RJ, Redfield MM: Contractility and ventricular systolic stiffening in hypertensive heart disease insights into the pathogenesis of heart failure with preserved ejection fraction. J Am Coll Cardiol 2009, 54:410-8.

23. Wang J, Kurrelmeyer KM, Torre-Amione G, Nagueh SF: Systolic and diastolic dyssynchrony in patients with diastolic heart failure and the effect of medical therapy. I Am Coll Cardiol 2007, 49:88-96.

24. Yu CM, Zhang Q, Yip GW, Lee PW, Kum LC, Lam YY, Fung JW: Diastolic and systolic asynchrony in patients with diastolic heart failure: a common but ignored condition. J Am Coll Cardiol 2007, 49:97-105

25. Westermann D, Kasner M, Steendijk P, Spillmann F, Riad A Weitmann K, Hoffmann W, Poller W, Pauschinger M, Schultheiss HP, Tschope C: Role of left ventricular stiffness in heart failure with normal ejection fraction. Circulation 2008, | | 7:205|-60.

FI000 Factor 3.0 Recommended

Evaluated by Michael Frenneaux 18 Jun 2008

26. Wachter R, Schmidt-Schweda S, Westermann D, Post H, Edelmann F, Kasner M, Luers C, Steendijk P, Hasenfuss G, Tschope C, Pieske B: Blunted frequency-dependent upregulation of cardiac output is related to impaired relaxation in diastolic heart failure. Eur Heart J 2009, 30:3027-36.

27. Martos R, Baugh J, Ledwidge M, O'Loughlin C, Conlon C, Patle A Donnelly SC, McDonald K: Diastolic heart failure: evidence of increased myocardial collagen turnover linked to diastolic dysfunction. Circulation 2007, I I 5:888-95.

28. Phan TT, Shivu GN, Abozguia K, Gnanadevan M, Ahmed I, Frenneaux M: Left ventricular torsion and strain patterns in heart failure with normal ejection fraction are similar to agerelated changes. Eur J Echocardiogr 2009, 1 0:793-800.

29. Tan YT, Wenzelburger F, Lee E, Heatlie G, Leyva F, Patel K, Frenneaux M, Sanderson JE: The pathophysiology of heart failure with normal ejection fraction: exercise echocardiography reveals complex abnormalities of both systolic and diastolic ventricular function involving torsion, untwist, and longitudinal motion. J Am Coll Cardiol 2009, 54:36-46.

30. Phan TT, Abozguia K, Nallur SG, Mahadevan G, Ahmed I, Williams L, Dwivedi G, Patel K, Steendijk P, Ashrafian H, Henning A, Frenneaux M: Heart failure with preserved ejection fraction is characterized by dynamic impairment of active relaxation and contraction of the left ventricle on exercise and associated with myocardial energy deficiency. J Am Coll Cardiol 2009, 54:402-9.

31. Phan TT, Abozguia K, Nallur SG, Ahmed I, Leyva F, Patel K, Frenneaux $\mathrm{M}$ : Increased atrial contribution to left ventricular filling compensates for impaired early filling during exercise in heart failure with preserved ejection fraction. J Card Fail 2009, 15:890-7.

32. Esposito A, De Cobelli F, Perseghin G, Pieroni M, Belloni E, Mellone R, Canu T, Gentinetta F, Scifo P, Chimenti C: Impaired left ventricular energy metabolism in patients with hypertrophic cardiomyopathy is related to the extension of fibrosis at delayed gadolinium-enhanced magnetic resonance imaging. BMJ 2009, 95:228.

33. Abozguia K, Nallur Shivu G, Phan TT, Ahmed I, Maher AR, Frenneaux M: Potential of metabolic agents as adjunct therapies in heart failure. Future Cardiol 2007, 3:525-35. 